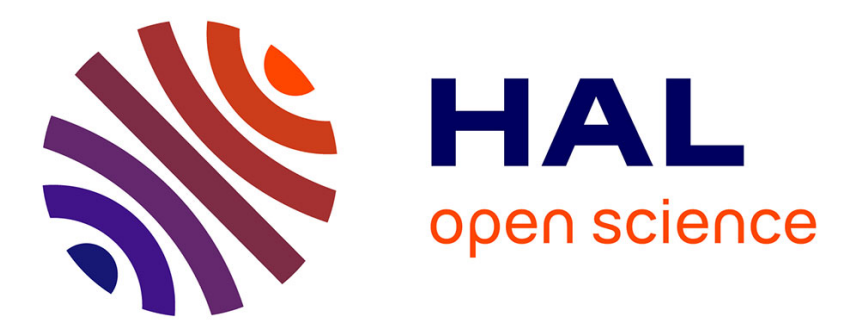

\title{
Automatic fire pixel detection using image processing: A comparative analysis of Rule-based and Machine Learning-based methods
}

\author{
Tom Toulouse, Lucile Rossi, Turgay Celik, Moulay Akhloufi
}

\section{- To cite this version:}

Tom Toulouse, Lucile Rossi, Turgay Celik, Moulay Akhloufi. Automatic fire pixel detection using image processing: A comparative analysis of Rule-based and Machine Learning-based methods. Signal, Image and Video Processing, 2015, pp.1863-1703. 10.1007/s11760-015-0789-x . hal-01181626

\author{
HAL Id: hal-01181626 \\ https://hal.science/hal-01181626
}

Submitted on $30 \mathrm{Jul} 2015$

HAL is a multi-disciplinary open access archive for the deposit and dissemination of scientific research documents, whether they are published or not. The documents may come from teaching and research institutions in France or abroad, or from public or private research centers.
L'archive ouverte pluridisciplinaire HAL, est destinée au dépôt et à la diffusion de documents scientifiques de niveau recherche, publiés ou non, émanant des établissements d'enseignement et de recherche français ou étrangers, des laboratoires publics ou privés. 


\title{
Automatic fire pixel detection using image processing: A comparative analysis of Rule-based and Machine Learning-based methods
}

\author{
Tom Toulouse · Lucile Rossi - Turgay Celik - Moulay Akhloufi
}

Received: 12.03.15 / Accepted: 11.06.15

\begin{abstract}
This paper ${ }^{1}$ presents a comparative analysis of state of the art image processing based fire color detection rules and methods in the context of geometrical characteristics measurement of wildland fires. Two new rules and two new detection methods using an intelligent combination of the rules are presented and their performances are compared with their counterparts. The benchmark is performed on approximately two hundred million fire pixels and seven hundred million non-fire pixels extracted from five hundred wildland images under diverse imaging conditions. The fire pixels are categorized according to fire color and existence of smoke, meanwhile, non-fire pixels are categorized according to the average intensity of the corresponding image. This characterization allows to analyze the performance of each rule by category. It is shown that the performances of the existing rules and methods from the literature are category dependent and none of them is able to perform equally well on all categories. Meanwhile, a new proposed method based on machine learning techniques and using all the rules as features outperforms existing state of the art techniques in the literature by performing almost equally well on different categories. Thus, this method, promises very interesting de-
\end{abstract}

T. Toulouse $\cdot$ L. Rossi

UMR CNRS 6134 SPE, University of Corsica, 20250 Corte, France

E-mail: toulouse@univ-corse.fr, lrossi@univ-corse.fr

T. Celik

School of Computer Science, University of the Witwatersrand, Johannesburg, South Africa and Electrical and Electronics Engineering, Meliksah University, Kayseri, Turkey

E-mail: celikturgay@gmail.com

M. Akhloufi

Department of Electronics Engineering, Universidad Tecnica Federico

Santa Maria, Valparaso, Chile

E-mail: akhloufi@gel.ulaval.ca velopments for the future of metrologic tools for fire detection in unstructured environments.

Keywords Fire pixel detection · Rules · Machine Learning · Wildland fire

\section{Introduction}

Wildland fires are responsible for serious damages. They cause deforestation and desertification, air pollution, economic losses and loss of lives among the public and firefighters. The fight against this major risk is all the more effective when it is possible to anticipate its behavior over time. Geometrical characteristics like position, rate of spread, length, surface, volume are needed to understand and model the phenomena occurring during fire propagation [10,20]. In the last 10 years, frameworks using cameras have been developed in order to be used as complementary metrological instrument in fire experiments [17]. The first and most important step for computer vision processing is the fire pixel detection because it determines the accuracy of the following treatments. In the visible spectrum, the main difficulties encountered by the detection methods are due to the fire color and the smoke. Indeed, the color can be inhomogeneous and different (depending on the background and the luminosity); the smoke can superimpose the fire areas.

Several fire detection algorithms are proposed in the literature [4]. In the visible spectrum, the algorithms often use color rules in different color spaces $R G B$ [19,24,3,14], $Y C_{b} C_{r}$ [2], YUV [16], $L^{*} a^{*} b^{*}$ [1], HSI [13], HSV [15] or combination of different color spaces [6,5,22]. Most of these works are used in an early fire detection context, were the real time performance is very important but in this paper, we are in-

1 The final publication is available at Springer via http://dx. doi.org/10.1007/s11760-015-0789-x 
terested in the analysis of the performance of fire pixel detection algorithms.

In the context of wildland fires, two studies [8,23] were carried out comparing the efficiency of Chen [6], Celik [2], Chitade [7], Ko [14] and Rossi [22] methods. Each study was done using a different set of images and the maximum number of pictures in the set was seventy six. This small number of images and the lack of information concerning the fire images does not allow to select the best color space and rules to detect wildland fire pixels.

Most of fire color detection methods use combination of rules on a color space because it is a fast and simple way to detect fire pixels on images. In order to determine which fire detection methods are more appropriate for wildland fire detection and how their performances depend on fire characteristics (fire color, brightness, presence of smoke), an analysis of the performances of the fire detection methods from the literature tested in a large number of outdoor vegetation fire images is necessary. Also, how the rules should be associated in order to obtain a better fire detection method deserves to be studied.

This article emphasizes on color spaces and rules used in fire pixel detection methods. It presents twenty nine wellknown color rules from the fire color detection literature. As these rules are not efficient on all type of images, this paper proposes two new rules to perform a better rule-based fire pixel detection. Two new detection algorithms that use the studied rules are also presented in this paper. One method combines rules with the same weight and the other uses machine learning with all the rules as input. For the first time, a fire pixel detection method that combines weighted rules using machine learning is presented. A benchmark of the all rules and detection algorithms is performed on a set of nine hundred millions labeled pixels and the results are presented for each image category.

The paper is organized as follows: Section 2 describes the state of the art fire pixel detection rules and the new proposed ones. Section 3 describes two new fire detection methods. Section 4 presents the image dataset and section 5 presents the performances of the new detection rules and all the detection methods. Section 6 concludes this paper.

\section{Fire pixel detection rules}

In general, each fire pixel detection method can be considered as logical combination of basic units; rules. Rules are composed of basic mathematical operations. In this section, twenty nine rules found in the literature of fire color detection methods and two new rules are described.

The following notation is used, unless otherwise stated: $I_{c}(\mathbf{x})$ is the intensity value of a pixel located at spatial location $(\mathbf{x})$ in color channel $c$ of the corresponding color space.
All rules are defined with the following format:

$r_{k}(\mathbf{x})=f_{k}(\mathbf{x})-c_{k}(\mathbf{x})$

where $r_{k}(\mathbf{x})$ is the rule $k, f_{k}(\mathbf{x})$ is the functional form of the rule and $c_{k}(\mathbf{x})$ is a constant. A pixel $\mathbf{x}$ of an image is labeled as fire if it verifies the following equation

$r_{k}(\mathbf{x}) \geq 0$

The definition of rule given in Eqn. (1) allows to fairly compare different schemes on the same dataset by learning the corresponding constants on a common training set through maximizing a performance metric. In this article, a training set of one million pixels extracted from a dataset of five hundred images is used. The $F$-score [9] is used as a performance metric to evaluate detection methods and rules. Given a detection method and a ground truth, there are four possible outcomes. If the ground truth of the pixel $\mathbf{x}$ is fire and it is detected as fire, it is counted as a true positive (TP); if it is detected as non-fire, it is counted as a false negative $(\mathrm{FN})$. If the ground truth of the pixel $\mathbf{x}$ is non-fire and it is detected as non-fire, it is counted as a true negative (TN); if it is detected as fire, it is counted as a false positive (FP). The detector performance is quantitatively measured using $F$-score according to:

$F=2 \cdot \frac{\text { Precision } \cdot \text { Recall }}{\text { Precision }+ \text { Recall }}$,

where

Precision $=\frac{\mathrm{TP}}{\mathrm{TP}+\mathrm{FP}}$, Recall $=\frac{\mathrm{TP}}{\mathrm{TP}+\mathrm{FN}}$.

The higher is the value of $F$-score the better is the performance.

\subsection{Fire pixel detection rules from the litterature}

Phillips et al. [19] proposed a supervised learning-based method which builds a 3D histogram from labeled fire images in $R G B$ color space. The $256 \times 256 \times 256$ Gaussian smoothing histogram is computed for the $R G B$ color space as follows: for each fire pixel of the image, a 3D Gaussian distribution with a standard deviation $\sigma=2$ is added to the color histogram centered in the pixel $(R, G, B)$ value and for each background pixel a 3D Gaussian distribution with $\sigma=1$ is subtracted from the color histogram. Then the values of the Gaussian histogram have positive values for colors that are probably fire and negative values for background colors. Let $H(\mathbf{x})$ be the value of the Gaussian smoothed histogram for the $R G B$ value of pixel $\mathbf{x}$. Then, the Phillips' detection can be expressed as the following rule

$r_{1}(\mathbf{x})=H(\mathbf{x})$. 
Chen et al. [6] have developed a fire detection procedure based on $R, G, B$ color channels and saturation $(S)$ values. It is a fast and efficient method and the rules have been reused in other fire detection methods [11]. The first two rules are for a rapid detection of pixels of color in the range of red to yellow. Red channel of fire pixels should be higher than their green channel and their green channel should be higher than their blue channel, i.e.,

$r_{2}(\mathbf{x})=I_{R}(\mathbf{x})-I_{G}(\mathbf{x})$,

$r_{3}(\mathbf{x})=I_{G}(\mathbf{x})-I_{B}(\mathbf{x})$.

As the fire is a light source, fire pixels are expected to have high red values, i.e.,

$r_{4}(\mathbf{x})=I_{R}(\mathbf{x})-\tau_{R}$,

where $\tau_{R}$ is a threshold. The fourth rule of Chen et al. uses the saturation value of pixels in order to eliminate some fire like regions due to background illumination, i.e.,

$r_{5}(\mathbf{x})=I_{S}(\mathbf{x})-\left(255-I_{R}(\mathbf{x})\right) \frac{\tau_{S}}{\tau_{R}}$,

where $\tau_{S}$ is a threshold. In [6], the two thresholds $\tau_{R}$ and $\tau_{S}$ are not specified. In our study, they have been estimated from our dataset using a pattern search optimization procedure [12]. This procedure computes the values $\tau_{R}$ and $\tau_{S}$ that maximize the mean $F$-score of combination of the rules $r_{2}$ to $r_{5}$ on the learning dataset. The thresholds $\tau_{R}=135$ and $\tau_{S}=53$ give the best results.

Horng et al. [13] proposed a rule-based detection method in HSI color space. Rules on $H, S$ and $I$ channel values are defined to classify a pixel as belonging to the fire. The following three rules are introduced,

$r_{6}(\mathbf{x})=-\left|I_{H}(\mathbf{x})-\tau_{H 1}\right|+\tau_{H 2}$,

$r_{7}(\mathbf{x})=-\left|I_{S}(\mathbf{x})-\tau_{S 1}\right|+\tau_{S 2}$,

$r_{8}(\mathbf{x})=-\left|I_{I}(\mathbf{x})-\tau_{I 1}\right|+\tau_{I 2}$,

where $\tau_{H 1}, \tau_{H 2}, \tau_{S 1}, \tau_{S 2}, \tau_{I 1}$ and $\tau_{I 2}$ are threshold values. In order to find these values, Horng et al. use $H, S$ and $I$ histograms of 70 flame images. In our study the training set were used to compute the histograms and it was found that the fire pixels have $H$ values (respectively $S$ and $I$ ) in [-45, 65] (respectively in [17, 200] and [35, 215]). The thresholds corresponding to these ranges are $\tau_{H 1}=10, \tau_{H 2}=55, \tau_{S 1}=$ 108.5, $\tau_{S 2}=91.5, \tau_{I 1}=125$ and $\tau_{I 1}=90$.

Celik et al. [3] developed six rules in $R G B$ color space. They first used the first two rules of Chen et al. and instead of rule $r_{4}$, they proposed to use the following rule:

$r_{9}(\mathbf{x})=I_{R}(\mathbf{x})-R_{\text {Mean }}$,

where $R_{\text {Mean }}$ is the mean of pixel values in $R$ channel. As the illumination of the scene is related to the luminance and chrominance changes in an image, the authors proposed three additional rules using $R G B$ ratios that represent at the same time the chrominance and the luminance:

$r_{10}(\mathbf{x})=-\left|\frac{I_{G}(\mathbf{x})}{I_{R}(\mathbf{x})+1}-\tau_{C 1_{1}}\right|+\tau_{C 1_{2}}$,

$r_{11}(\mathbf{x})=-\left|\frac{I_{B}(\mathbf{x})}{I_{R}(\mathbf{x})+1}-\tau_{C 2_{1}}\right|+\tau_{C 2_{2}}$,

$r_{12}(\mathbf{x})=-\left|\frac{I_{B}(\mathbf{x})}{I_{G}(\mathbf{x})+1}-\tau_{C 3_{1}}\right|+\tau_{C 3_{2}}$,

where the thresholds are chosen by Celik et al. thanks to observation on the values of channels ratios on hundred fifty fire images from Internet. Using the same method than before, the thresholds corresponding to our dataset are obtained as $\tau_{C 1_{1}}=0.565, \tau_{C 1_{2}}=0.315, \tau_{C 2_{1}}=0.415, \tau_{C 2_{2}}=$ $0.415, \tau_{C 3_{1}}=0.08, \tau_{C 3_{2}}=-0.08$.

Ko et al. [14] proposed a probabilistic approach to detect fire in $R G B$ color space. $R, G, B$ color channels of fire pixels are assumed to be independent and each channel is modeled with a unimodal Gaussian distribution, i.e.,

$p_{c}\left(I_{c}(\mathbf{x})\right)=\frac{1}{\sqrt{2 \pi \sigma_{c}^{2}}} \exp \left(-\frac{\left(I_{c}(\mathbf{x})-\mu_{c}\right)^{2}}{2 \sigma_{c}^{2}}\right)$,

where $c \in\{R, G, B\}, \mu_{c}$ and $\sigma_{c}^{2}$, respectively, are the mean and variance of the data from colour channel $c$.

$p_{f}(\mathbf{x})=\prod_{c \in\{R, G, B\}} p_{c}\left(I_{c}(\mathbf{x})\right)$.

Using the above equation, the following rule is introduced

$r_{13}(\mathbf{x})=p_{f}(\mathbf{x})-\tau_{K}$,

where $\tau_{K}$ is a threshold value not specified in [14]. It is optimized in this study with pattern search on our dataset at $\tau=3.43 \times 10^{-5}$.

In [2], Celik et al. developed eight rules on $Y C_{b} C_{r}$ color space in order to alleviate the problems caused from illumination changes and to use the consistency in chromicity representation. The first two rules are the translation of the rules $r_{2}, r_{3}$ and $r_{9}$ into $Y C_{b} C_{r}$ color space, i.e.,

$r_{14}(\mathbf{x})=I_{Y}(\mathbf{x})-I_{C_{b}}(\mathbf{x})$,

$r_{15}(\mathbf{x})=I_{C_{r}}(\mathbf{x})-I_{C_{b}}(\mathbf{x})$.

For the next three rules, the mean of pixels intensity is used to segment the brighter region of the image, i.e.,

$r_{16}(\mathbf{x})=I_{Y}(\mathbf{x})-Y_{\text {Mean }}$,

$r_{17}(\mathbf{x})=C_{b_{\text {Mean }}}-I_{C_{b}}(\mathbf{x})$,

$r_{18}(\mathbf{x})=I_{C_{r}}(\mathbf{x})-C_{r_{\text {Mean }}}$,

where $Y_{\text {Mean }}, C_{b_{\text {Mean }}}$ and $C_{r_{\text {Mean }}}$ are the mean of pixel values in $Y, C_{b}$ and $C_{r}$ channels respectively. The next rule comes from the fact that in their observations, the authors noticed 
a significant difference between the $C_{b}$ and $C_{r}$ intensities of the fire pixels, i.e.,

$r_{19}(\mathbf{x})=\left|I_{C_{b}}(\mathbf{x})-I_{C_{r}}(\mathbf{x})\right|-\tau_{C}$,

where they optimized the threshold thanks to ROC curves analysis [9] on one thousand images. In our study, it has been optimized to $\tau_{C}=40$ with pattern search procedure. The last two rules are tied to the observation that the distribution of fire pixels on the $C_{B}-C_{r}$ plane is enclosed by curves, i.e.,

$r_{20}(\mathbf{x})=f_{1}\left(I_{C_{r}}(\mathbf{x})\right)-I_{C_{b}}(\mathbf{x})$,

$r_{21}(\mathbf{x})=I_{C_{b}}(\mathbf{x})-f_{2}\left(I_{C_{r}}(\mathbf{x})\right)$.

In our study the curves $f_{1}$ and $f_{2}$ are computed on the training set and defined as follow:

$f_{1}(x)=7.79 \times 10^{-3} x^{2}+2.10 x-2.25$,

$f_{2}(x)= \begin{cases}4.47 \times 10^{-2} x^{2}-16.94 x+1513.52 & \text { if } x \leq 142 \\ 3.39 \times 10^{-5} x^{2}+0.77 x-98.31 & \text { otherwise }\end{cases}$

Celik [1] proposed four detection rules based on the CIE $L^{*} a^{*} b^{*}$ colorspace. He assumed that the fire region is the brightest area in the image and has a color near the red color, the following rules are defined

$r_{22}(\mathbf{x})=I_{L^{*}}(\mathbf{x})-L_{M e a n}^{*}$,

$r_{23}(\mathbf{x})=I_{a^{*}}(\mathbf{x})-a_{\text {Mean }}^{*}$,

$r_{24}(\mathbf{x})=I_{b^{*}}(\mathbf{x})-b_{\text {Mean }}^{*}$

$r_{25}(\mathbf{x})=I_{b^{*}}(\mathbf{x})-I_{a^{*}}(\mathbf{x})$,

where $L_{\text {Mean }}^{*}, a_{\text {Mean }}^{*}$ and $b_{\text {Mean }}^{*}$ are the mean values of the intensity in the $L^{*}, a^{*}$ and $b^{*}$ color channels, respectively.

Chen et al. [5] studied fire pixel saturation. Thanks to this study, the authors introduced a color saturation constraint. Indeed, they observed that the fire pixels are in a bounded region enclosed by two curves, i.e.,

$r_{26}(\mathbf{x})=100 \times I_{S}(\mathbf{x})-f_{3}\left(I_{B}(\mathbf{x})\right)$,

$r_{27}(\mathbf{x})=f_{4}\left(I_{B}(\mathbf{x})\right)-100 \times I_{S}(\mathbf{x})$.

In our training set, the equations of the curves $f_{3}$ and $f_{4}$ are defined as follows

$f_{3}(x)=80 \exp \left(\frac{x}{25}\right)$,

$f_{4}(x)=6.5 \times 10^{-3} x^{2}-0.55 x+100$.

They also used the rules $r_{2}, r_{3}$ and $r_{4}$ in combination with $r_{26}$ and $r_{27}$ to detect fire on images.

Rossi and Akhloufi [21] proposed a method that combines information from $R G B$ and $Y U V$ color spaces. The $k$ means clustering technique with $k=2$ is applied to $V$ channel of $Y U V$ color space in order to separate pixels into lowintensity and high-intensity classes. This operation is similar to a threshold on the $V$ channel. In order to study it in this paper, the rule is expressed as follow:

$r_{28}(\mathbf{x})=I_{V}(\mathbf{x})-\tau_{V}$,

where $\tau_{V}$ is computed with the Otsu method [18]. Pixels selected with this rule are further analyzed according to a fire color model defined in $R G B$ color space. Fire color model is represented with 3D Gaussian model and corresponding model parameters are obtained from a labeled dataset. The second rule is then defined as follows

$r_{29}(\mathbf{x})=-\sqrt{\sum_{C \in\{R, G, B\}}\left(I_{C}(\mathbf{x})-m_{C}\right)^{2}}+\tau_{\sigma} * \sigma$,

where $m_{C}$ is the mean of the channel $C$ for pixels that verify the rule $r_{28}$ and $\sigma=\max _{\{R, G, B\}}\left(\sigma_{C}\right)$ where $\sigma_{C}$ is the standard deviation of the channel $C$ for pixel that verify the rule $r_{28}$. The threshold $\tau_{\sigma}$ is not specified in [21]. In this study, the threshold is optimized to $\tau_{\sigma}=2.5$ with a pattern search procedure on the learning set of pixels.

\subsection{New fire pixel detection rules}

The rules presented in the previous section are not always efficient for all types of fire pixels. In the following we present two new rules that obtain better performances.

The first rule uses $a^{*}$ and $b^{*}$ channels from the $L^{*} a^{*} b^{*}$ color space. Red and orange fire regions are clearly separable in the $a^{*}+b^{*}$ images even in presence of smoke. This demarcation comes from the fact that positive values of $a^{*}$ channel represent shades of red and positive values of $b^{*}$ channel represent shades of yellow which is represented as new rule as follows:

$r_{30}(\mathbf{x})=I_{a^{*}}(\mathbf{x})+I_{b^{*}}(\mathbf{x})-t_{1}$,

where $t_{1}=32.4$ is a threshold obtained using a pattern search procedure on the learning set.

The second rule uses the $R G B$ colorspace and the fact that for reddish and orange colors, the channel $R$ is much higher than $G$ or $B$ channels. The rule identifies fire pixels when the difference between their red value and green or blue value is higher than a threshold $t_{2}$, i.e.,

$r_{31}=I_{R}(\mathbf{x})-\min \left(I_{R}(\mathbf{x}), I_{G}(\mathbf{x}), I_{B}(\mathbf{x})\right)-t_{2}$,

where $t_{2}=72$ is a threshold obtained using the same method that was used to compute $t_{1}$. 


\section{Expert systems}

A combination of rules using different color spaces may give a better score than that achieved by individual rules but the difficulty is to choose the rules to be combined and their weights in association. In this section, two new fire pixel detection methods based on combination of rules are presented. The first method uses a majority voting of all rules. The second method uses machine learning, specifically logistic regression, to combine rules.

\subsection{Majority voting}

The majority voting considers outputs of all rules to produce a final output. Thus, if a pixel verifies a certain number of rules, it is considered as a fire pixel. So a pixel is labeled fire if the following condition is verified

$$
\left(\sum_{k=1}^{31} \varphi_{k}(\mathbf{x})\right) \geq \tau_{M V}
$$

were $\varphi_{k}$ is defined as follows

$\varphi_{k}(\mathbf{x})=\left\{\begin{array}{ll}1 & \text { if } r_{k} \geq 0 \\ 0 & \text { otherwise }\end{array}\right.$,

and $\tau_{M V}$ is a threshold value. In order to find $\tau_{M V}$, all values of $\tau_{M V}$ in $[1,31]$ are tested using the $F$-score. In our training set, the best F-score is obtained with $\tau_{M V}=23$.

\subsection{Learning from rules using logistic regression}

Logistic regression is a probabilistic classification method. The classifier learns a hypothesis $h_{\mathbf{w}}$ that is parameterized by $\mathbf{w} \in \mathbb{R}^{(d+1) \times 1}$ for feature vector $\mathbf{x} \in \mathbb{R}^{(d+1) \times 1}$, i.e.,

$h_{\mathbf{w}}(\mathbf{x})=\frac{1}{1+e^{-\mathbf{w}^{T} \mathbf{x}}}$,

where $(\cdot)^{T}$ is vector transpose, $\mathbf{w}=\left[w_{0}, \cdots, w_{d}\right]^{T}, \mathbf{x}=\left[x_{0}\right.$, $\left.\cdots, x_{d}\right]^{T}, x_{0}=1, x_{k}=r_{k}, k=1, \ldots, d$, are specific features and $h_{\mathbf{w}}(\mathbf{x}) \in[0,1]$.

The parameters of $h_{\mathbf{w}}$ are learned using a training set $\left\{\left(\mathbf{x}^{(i)}, y^{(i)}\right)\right\}_{i=1}^{N}$ of size $N$, where each training sample $\left(\mathbf{x}^{(i)}, y^{(i)}\right)$ represents the corresponding feature vector $\mathbf{x}^{(i)}$ with a label $y^{(i)}$ of fire $\left(y^{(i)}=1\right)$ or non-fire $\left(y^{(i)}=0\right)$. The parameters are iteratively learned by minimizing a mean square error $(J(\mathbf{w}))$ between the real labels $y^{(i)}$ s and the predicted labels $h_{\mathbf{w}}\left(\mathbf{x}^{(i)}\right)$ s resulting from the regression, i.e.,

$J(\mathbf{w})=\frac{1}{N} \sum_{i=1}^{N}\left(y^{(i)}-h_{\mathbf{w}}\left(\mathbf{x}^{(i)}\right)\right)^{2}$.
As a minimizer of the mean square error function, gradient descent algorithm is employed to obtain the following iterative equations:

$w_{k} \leftarrow w_{k}-\alpha \frac{1}{N} \sum_{i \in N} \delta^{(i)}$,

where

$\delta^{(i)}=h_{\mathbf{w}}\left(\mathbf{x}^{(i)}\right)-\mathbf{y}^{(i)}$,

and $\alpha \in(0,1]$ stands for the learning rate.

Since, by definition, logistic regression trains a hypothesis function which is the sigmoid function, the result of the logistic regression is a continuous value bounded to the interval $[0,1]$. Thus, it produces a likelihood $h_{\mathbf{w}}(\mathbf{x})$ that a feature vector $\mathbf{x}$ is a fire feature vector. In other words, one can threshold the hypothesis $h_{\mathbf{w}}(\mathbf{x})$ according to $h_{\mathbf{w}}(\mathbf{x})>T_{h}$ to obtain a crisp value that the corresponding feature vector is a fire feature vector or not. The threshold $T_{h}=0.5$ is used in experiments, but a different value can be used.

In order to learn from rules, the rules defined in previous sections are used as feature values, i.e.,

$x_{k}=r_{k}$,

and the corresponding parameter set of the hypothesis is obtained from the training set of pixels.

\section{Image dataset}

A set of five hundred $R G B$ images of outdoor vegetation fires at different sizes (from $183 \times 242$ pixels to $4000 \times 3000$ pixels) and different image formats (jpg, ppm, bmp) has been created. The dataset is composed of free images collected from Internet and images from firefighters and researchers taken during real fires and experiments. The images were selected in order to represent the largest number of different fire contexts with heterogeneous environment (forest, maquis shrubland, rocks, snow,...) and different luminosity characteristics (sunny, cloudy, gray sky, blue sky, night, day,...). Figure 1 shows example images from the dataset.

For each image, the fire area was manually segmented by an expert. This region is called ground truth. Using HSI color space, the fire pixels are labeled in color type which can be red $\left(-57^{\circ}<I_{H}(\mathbf{x})<14^{\circ}\right.$ and $\left.50<I_{I}(\mathbf{x})<200\right)$, orange $\left(14^{\circ}<I_{H}(\mathbf{x})<42^{\circ}\right.$ and $\left.50<I_{I}(\mathbf{x})<200\right)$, whiteyellow $\left(42^{\circ}<I_{H}(\mathbf{x})<64^{\circ}\right.$ and $50<I_{I}(\mathbf{x})$, or $\left.200<I_{I}(\mathbf{x})\right)$ or other color for the other cases. A learning procedure using Support Vector Machine is used to classify pixels as "smoke" and "smokeless". The brightness of background pixels is obtained by analyzing the $I$ channel of the HSI color space. Three levels of intensity can be assigned to a pixel: "high intensity" $\left(115<I_{I}(\mathbf{x})\right)$, "medium intensity" $(50<$ 


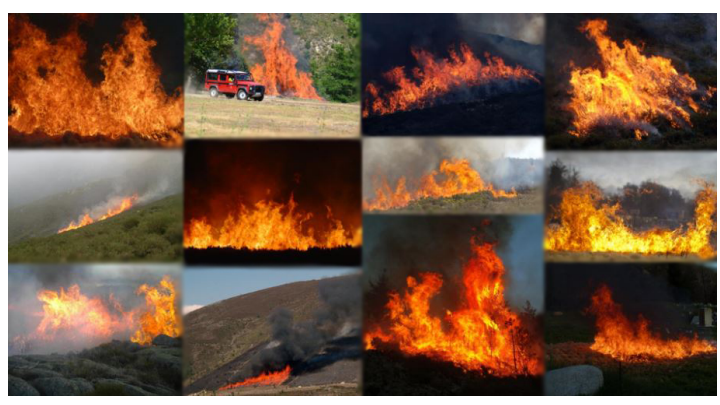

Fig. 1 Example of images from the dataset.

\begin{tabular}{|c|c|c|c|c|}
\hline & & & Number of pixels & Percentage \\
\hline \multirow{9}{*}{$\begin{array}{l}\frac{n}{0} \\
\stackrel{凶}{0} \\
\stackrel{0}{0}\end{array}$} & \multirow{2}{*}{ Red } & Smoke & 19334066 & $8.1 \%$ \\
\hline & & No Smoke & 43373644 & $18.2 \%$ \\
\hline & \multirow{2}{*}{ Orange } & Smoke & 32812298 & $13.8 \%$ \\
\hline & & No Smoke & 115227509 & $48.4 \%$ \\
\hline & \multirow{2}{*}{$\begin{array}{l}\text { White- } \\
\text { Yellow }\end{array}$} & Smoke & 18519389 & $7.8 \%$ \\
\hline & & No Smoke & 2426830 & $1.0 \%$ \\
\hline & \multirow{2}{*}{ Other } & Smoke & 578832 & $0.2 \%$ \\
\hline & & No Smoke & 5332683 & $2.2 \%$ \\
\hline & & All & 237950619 & $100 \%$ \\
\hline \multirow{4}{*}{ 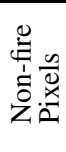 } & \multicolumn{2}{|c|}{ Low Intensity } & 170088686 & $21.9 \%$ \\
\hline & \multicolumn{2}{|c|}{ Medium Intensity } & 259874877 & $33.5 \%$ \\
\hline & \multicolumn{2}{|c|}{ High Intensity } & 345107529 & $44.5 \%$ \\
\hline & \multicolumn{2}{|c|}{ All } & 775071092 & $100 \%$ \\
\hline
\end{tabular}

Table 1 Distribution of the dataset pixels by category

$\left.I_{I}(\mathbf{x})<115\right)$ and "low intensity" $\left(I_{I}(\mathbf{x})<50\right)$. Table 1 gives the pixel distribution by category in the dataset. This distribution shows that the fire pixels are in majority orange and that the fire pixel labeled "other" can be ignored due to the low percentage of these pixels.

In order to build a learning set of pixels, fifty images are chosen randomly among the five hundred images. The fire pixels of these fifty images are sorted in six categories depending to the color of the pixels (red, orange, white-yellow) and the presence of smoke. The non fire pixels are classified using three levels of intensity (low medium and high). 500 000 non fire pixels and 500000 fire pixels are chosen in the following way. For each category, each pixel is represented with a feature vector constructed from color features from different color spaces. An average feature vector is computed for each category and the pixels are sorted according to the distance of their feature vectors to the average feature vector. The pixels are then sampled uniformly to obtain the desired number for each category. According to the observations of the Table 1, the pixel distribution is done as follows: $50 \%$ of orange pixel, $33 \%$ of red pixel and $17 \%$ of whiteyellow pixel for fire pixels and $20 \%$ of low intensity pixel, $40 \%$ of medium intensity pixels and $40 \%$ of high intensity pixel for non-fire pixels. This set has been used to compute the functions and constants for the rules and also to train the logical regression based approach. The fifty images used to build the learning set are not included in the testing set which therefore contains four hundred fifty images.

\section{Performances evaluation}

\subsection{Performances of rules}

In this section a comparative analysis of thirty one rules on the pixels of the testing image set is presented. The performances of rules are analyzed by pixel category. In our testing set there are approximately two hundred twelve millions fire pixels and six hundred ninety one millions non-fire pixels. Every fire pixel is categorized according to the categorization given in the Table 1 except for the "other" color category which is ignored. So the fire pixels can be regrouped in six groups and the non-fire pixels in three groups. Every rule is then evaluated on the types of pixels, computing the true positive and false negative pixels for fire pixels and false positive and true negative pixels for non-fire pixels. The table 2 presents the true positives (TP) and true negatives (TN) percentages for all the rules in each pixel category. For a better readability, scores are presented in shades of gray where white correspond to the best score. The first six rows of the table represent the true positives according to the cathegory of the fire pixels and the 3 next row are the true negatives according to the cathegory of non-fire pixels

The rule $r_{1}$ seems to be very efficient as it has high TP rates and high TN rates. It is less efficient for detecting white-yellow pixels, because of their similarity to sky pixels.

The rules $r_{2}, r_{3}, r_{4}$, and $r_{5}$ detect well the fire pixels except for red pixel for the rules $r_{3}, r_{5}$ and even for the rule $r_{4}$ that work on reddish color. But these rules give a low ratio of TN pixels, except for the rule $r_{4}$ which high ratio of TN pixels for low and medium intensity.

The rules $r_{6}, r_{7}$, and $r_{8}$ have high TP rates but also low TN rates. This is due to the fact that the thresholds were selected in order to have a maximum of fire pixel in the intervals, and that the intervals are wide due to the diversity of images.

The rules $r_{9}, r_{10}, r_{11}$, and $r_{12}$ have high TP rates except for yellow color fires for rules $r_{10}$ and $r_{12}$ but they have low TN rates except for high intensity pixels for rules $r_{10}$ and $r_{11}$. The rule $r_{9}$ has high TP rates and a high TN rates for medium and low intensity. This shows its efficiency detecting fires in low or medium intensity environments. It should be noted that the threshold of rule $r_{9}$ is dependent to the image characteristics. Rule $r_{12}$ has very lower TN rates compared to its good TP rates in general. This last rule is not suitable for fire segmentation.

Rule $r_{13}$ does not seem to be appropriate to fire detection. Indeed, it has a very low TP rates even if its $\mathrm{TN}$ rates are good.

The rules $r_{14}, r_{15}, r_{16}, r_{17}, r_{18}, r_{19}, r_{20}$, and $r_{21}$ have high TP 
Table 2 True negatives and true positives Scores of fire segmentation methods. The whiteness of the boxes indicates the efficiency

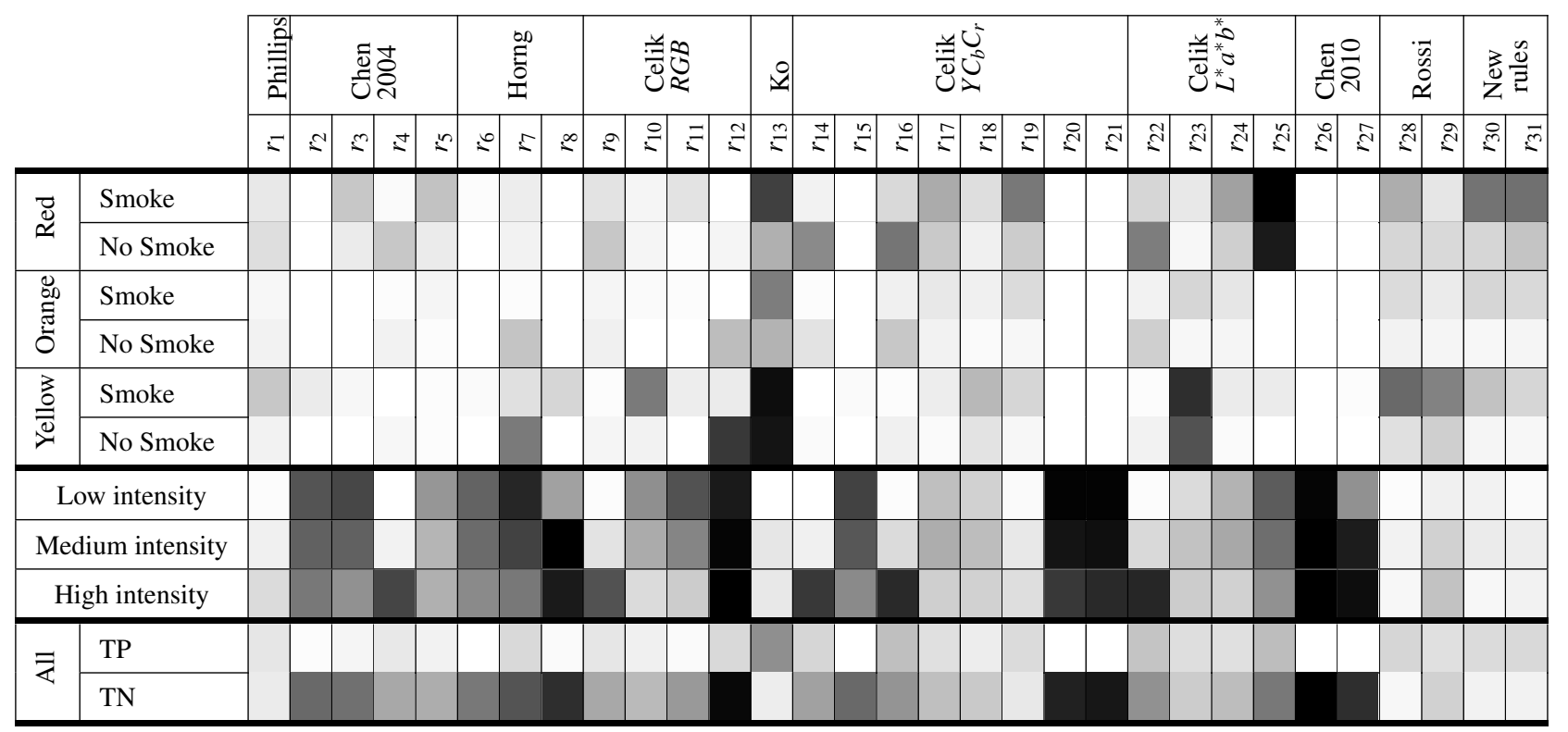

rates for orange and yellow colors but rules $r_{14}, r_{16}$, and $r_{19}$ have lower rated for reddish colors. The rules $r_{17}, r_{18}$, and $r_{19}$ have good TN rate. The rules $r_{20}$, and $r_{21}$ (using pixel distributions enclosed by curves in two color channels plane) have low TN values. This is due to the fact that they select non-only fire pixels but also a lot of environment pixels.

For the rules $r_{22}, r_{23}, r_{24}$, and $r_{25}$, there is a high TP rates for orange pixels and also for yellow pixels for rules $r_{22}, r_{24}$ and $r_{25}$.

The rules $r_{26}$ and $r_{27}$ give too low TN rates. The rule $r_{26}$ selects approximatively all pixels (fire and non-fire). This rule is not a suitable option for file detection. The rule $r_{27}$ removes only $11 \%$ of background pixel of medium intensities and 5\% for high intensity, making it less interesting for an efficient detection of fire pixels.

The rules $r_{28}$ and $r_{29}$ give better result for fire pixels without smoke, especially for orange colors. The rates of TN are very high for the rule $r_{28}$.

The new rules are also more efficient on fire without smoke, especially for orange pixel and have very high TN rates.

\subsection{Performances of the proposed methods}

The new proposed two methods are evaluated and compared with the other methods presented in this paper. The results are shown in the Table 3 . The scores obtained are dependent of the pixel categories. Among the methods from the literature, Phillips et al. gives the best result and is robust to smoke. The Chen 2004 and Chen 2010 methods give good scores for orange and yellow pixels. Celik $Y C_{b} C_{r}$ and Celik $L^{*} a^{*} b^{*}$ methods obtain high scores for orange pixels. Rossi method is efficient for fire pixels without smoke and particularly for orange pixels.

The proposed methods perform equally well on different categories and gives comparable results. The logistic regression method using rules as features is the overall best performing method. It gives the best results for nine categories of pixels and get high scores for the others. It is indeed robust to smoke and color changes.

\section{Conclusion}

In this paper, twenty nine rules of the literature were presented and two new rules were introduced for wildland fire color pixel detection. A dataset of five hundred fire images is used to compare these rules. More than two hundred thirty seven millions of fire pixels are classified according to their color and to the presence of smoke and more than seven hundred seventy five millions of background pixels are classified according to their luminosity. This categorization is used to show the specific effect of rules on the different types of pixels. The analysis of the performances showed the efficiency of the rules of Phillips et al. , Rossi et al. and the new proposed rules. Two different ways to combine the rules for fire pixels detection were also proposed. One of the new methods introduced here uses a machine learning technique involving logistic regression. This method obtains the overall best performance compared to all the state of the art benchmarked techniques. It is also robust to color changes and the presence of smoke. The obtained results are promising and show the possibility of developing more robust fire detection techniques in unstructured environments. 
Table 3 Scores of fire segmentation methods with the same weight for fire and non fire pixels

\begin{tabular}{|c|c|c|c|c|c|c|c|c|c|c|c|c|c|}
\hline & & & 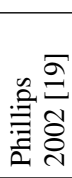 & $\begin{array}{r}\sigma \\
\text { పేరి } \\
\text { ర్ల }\end{array}$ & 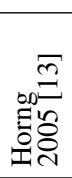 & 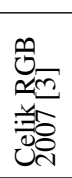 & 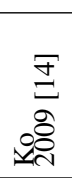 & 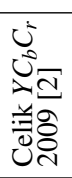 & 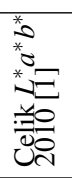 & 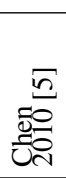 & 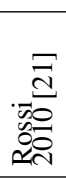 & 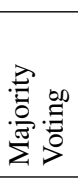 & 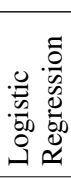 \\
\hline \multirow{6}{*}{$\begin{array}{l}\ddot{\mathscr{Q}} \\
\simeq\end{array}$} & \multirow{3}{*}{ 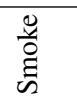 } & Low & 0.95 & 0.80 & 0.87 & 0.81 & 0.39 & 0.56 & 0.00 & 0.88 & 0.79 & $\begin{array}{l}0.93 \\
\end{array}$ & 0.97 \\
\hline & & Mediu & 0.92 & 0.78 & 0.78 & 0.79 & 0.37 & 0.56 & 0.00 & 0.85 & 0.78 & 0.88 & 0.92 \\
\hline & & High & 0.89 & 0.73 & 0.86 & 0.77 & 0.37 & 0.55 & 0.00 & 0.74 & 0.78 & 0.85 & 0.88 \\
\hline & \multirow{3}{*}{ 之o } & Low & 0.93 & 0.85 & 0.89 & 0.83 & 0.82 & 0.50 & 0.10 & 0.85 & 0.87 & 0.93 & 0.97 \\
\hline & & Mediu & 0.90 & 0.83 & 0.80 & 0.80 & 0.77 & 0.50 & 0.10 & 0.83 & 0.85 & 0.88 & 0.92 \\
\hline & & High & 0.87 & 0.78 & 0.87 & 0.78 & 0.78 & 0.49 & 0.10 & 0.72 & 0.86 & 0.86 & 0.88 \\
\hline \multirow{6}{*}{ 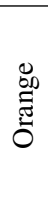 } & \multirow{3}{*}{$\begin{array}{l}\text { ஜै } \\
\text { है }\end{array}$} & Low & 0.98 & 0.98 & 0.90 & 0.97 & 0.66 & 0.89 & 0.86 & 1.00 & 0.90 & 0.98 & 0.98 \\
\hline & & Medium & 0.95 & 0.96 & 0.81 & 0.95 & 0.62 & 0.89 & 0.86 & 0.98 & 0.89 & 0.93 & 0.93 \\
\hline & & High & 0.92 & 0.91 & 0.89 & 0.93 & 0.62 & 0.87 & 0.84 & 0.86 & 0.89 & 0.90 & 0.89 \\
\hline & \multirow{3}{*}{$z^{\stackrel{y}{d}}$} & Low & 0.97 & 0.97 & 0.78 & 0.81 & 0.82 & 0.85 & 0.88 & 0.97 & 0.96 & 0.98 & 0.98 \\
\hline & & $\mathrm{M}$ & 0.94 & 0.95 & 0.69 & 0.79 & 0.78 & 0.85 & 0.88 & 0.95 & 0.94 & 0.92 & 0.93 \\
\hline & & High & 0.91 & 0.90 & 0.76 & 0.77 & 0.78 & 0.84 & 0.86 & 0.84 & 0.95 & 0.90 & 0.89 \\
\hline \multirow{6}{*}{$\frac{3}{\frac{a}{0}}$} & \multirow{3}{*}{$\begin{array}{l}\stackrel{\mathscr{y}}{0} \\
\stackrel{y}{5}\end{array}$} & Lov & 0.87 & 0.94 & 0.78 & 0.59 & 0.09 & 0.78 & 0.20 & 0.94 & 0.52 & 0.96 & 0.98 \\
\hline & & Medi & 0.85 & 0.92 & 0.69 & 0.57 & 0.09 & 0.77 & 0.20 & 0.92 & 0.51 & 0.91 & 0.93 \\
\hline & & High & 0.81 & 0.86 & 0.76 & 0.56 & 0.09 & 0.76 & 0.20 & 0.80 & 0.51 & 0.88 & 0.89 \\
\hline & \multirow{3}{*}{ zo } & Low & 0.97 & 0.98 & 0.58 & 0.25 & 0.14 & 0.92 & 0.47 & 0.98 & 0.88 & 0.97 & 0.97 \\
\hline & & Medi & 0.95 & 0.96 & 0.50 & 0.24 & 0.13 & 0.92 & 0.47 & 0.96 & 0.86 & 0.92 & 0.92 \\
\hline & & Hig & 0.91 & 0.91 & 0.56 & 0.24 & 0.13 & 0.90 & 0.46 & 0.84 & 0.87 & 0.89 & 0.88 \\
\hline & 0.91 & 0.88 & 0.78 & 0.79 & 0.68 & 0.76 & 0.67 & 0.86 & 0.88 & 0.90 & 0.91 \\
\hline
\end{tabular}

\section{References}

1. Celik, T.: Fast and efficient method for fire detection using image processing. ETRI journal 32(6), 881-890 (2010)

2. Celik, T., Demirel, H.: Fire detection in video sequences using a generic color model. Fire Safety Journal 44(2), 147158 (2009)

3. Celik, T., Demirel, H., Ozkaramanli, H., Uyguroglu, M.: Fire detection using statistical color model in video sequences. Journal of Visual Communication and Image Representation 18(2), 176185 (2007). DOI 10.1016/j.jvcir.2006.12.003

4. Çetin, A.E., Dimitropoulos, K., Gouverneur, B., Grammalidis, N., Günay, O., Habiboğlu, Y.H., Töreyin, B.U., Verstockt, S.: Video fire detectionreview. Digital Signal Processing 23(6), 18271843 (2013)

5. Chen, J., He, Y., Wang, J.: Multi-feature fusion based fast video flame detection. Building and Environment 45(5), 11131122 (2010). DOI 10.1016/j.buildenv.2009.10.017

6. Chen, T.H.,Wu, P.H., Chiou, Y.C.: An early fire-detection method based on image processing. In: Image Processing, ICIP04. International Conference on, vol. 3, pp. 17071710. IEEE (2004)

7. Chitade, A.Z., Katiyar, S.: Colour based image segmentation using $\mathrm{k}$-means clustering. International Journal of Engineering Science and Technology 2(10), 53195325 (2010)

8. Collumeau, J.F., Laurent, H., Hafiane, A., Chetehouna, K.: Fire scene segmentations for forest fire characterization: A comparative study. In: Image Processing (ICIP), 2011 18th IEEE International Conference on, pp. 29732976 (2011)

9. Fawcett, T.: Roc graphs: Notes and practical considerations for researchers. Machine learning 31, 138 (2004)

10. Grishin, A.M.: Mathematical modeling of forest fires and new methods of fighting them. Publishing house of the Tomsk state university (1997)

11. Habiboğlu, Y.H., Günay, O., Çetin, A.E.: Covariance matrix-based fire and flame detection method in video. Machine Vision and Applications 23(6), 11031113 (2011)

12. Hooke, R., Jeeves, T.A.: direct searchsolution of numerical and statistical problems. Journal of the ACM 8(2), 212229 (1961)
13. Horng, W.B., Peng, J.W., Chen, C.Y.: A new image-based realtime flame detection method using color analysis. In: Networking, Sensing and Control, Proceedings. IEEE, pp. 100105 (2005)

14. Ko, B.C., Cheong, K.H., Nam, J.Y.: Fire detection based on vision sensor and support vector machines. Fire Safety Journal 44(3), 322329 (2009)

15. Liu, C.B., Ahuja, N.: Vision based fire detection. In: Pattern Recognition, 2004. ICPR 2004. Proceedings of the 17th International Conference on, 4, pp. 134137. IEEE (2004)

16. Marbach, G., Loepfe, M., Brupbacher, T.: An image processing technique for fire detection in video images. Fire safety journal 41(4), 285289 (2006)

17. Martínez-de Dios, J.R., Merino, L., Caballero, F., Ollero, A.: Automatic forest-fire measuring using ground stations and unmanned aerial systems. Sensors 11(6), 63286353 (2011)

18. Otsu, N.: A threshold selection method from gray-level histograms. Automatica 11(285-296), 2327 (1975)

19. Phillips III, W., Shah, M., da Vitoria Lobo, N.: Flame recognition in video. Pattern recogn. Lett. 23(1), 319327 (2002)

20. Rossi, J.L., Chetehouna, K., Collin, A., Moretti, B., Balbi, J.H.: Simplified flame models and prediction of the thermal radiation emitted by a flame front in an outdoor fire. Combustion Science and Technology 182(10), 14571477 (2010)

21. Rossi, L., Akhloufi, M.: Dynamic fire 3D modeling using a realtime stereovision system. In: M. Iskander, V. Kapila, M.A. Karim (eds.) Technological Developments in Education and Automation, pp. 3338. Springer Netherlands, Dordrecht (2010)

22. Rossi, L., Akhloufi, M., Tison, Y.: On the use of stereovision to develop a novel instrumentation system to extract geometric fire fronts characteristics. Fire Safety Journal 46, 920 (2011)

23. Rudz, S., Chetehouna, K., Hafiane, A., Laurent, H., SeroGuillaume, O., : Investigation of a novel image segmentation method dedicated to forest fire applications. In: Measurement Science and Technology 24(7), pp.075403 (2013)

24. Töreyin, B.U., Dedeoğlu, Y., Güdükbay, U., Çetin, A.E.: Computer vision based method for real-time fire and flame detection. Pattern Recognition Letters 27(1), 4958 (2006) 